\title{
STRUCTURE AND BEHAVIOUR OF THE SPERM TERMINAL FILAMENT
}

\author{
C. K. OMOTO AND C. J. BROKAW \\ Division of Biology, California Institute of Technology, \\ Pasadena, $C A$ 91 125 , U.S.A.
}

\begin{abstract}
SU M M A R Y
Light- and electron-microscopic observations of Ciona and Lytechinus spermatozoa show a thin terminal filament at the distal end. The terminal filament is $5-6 \mu \mathrm{m}$ long and contains the two central microtubules and a variable number of A-tubule extensions of the peripheral doublet microtubules. The transition from the $9+2$ region to the terminal filament is tapered more gradually in Lytechinus than in Ciona. Photographs of the movement of beating spermatozoa do not show any obvious discontinuity in curvature at the transition region. Bends are propagated smoothly off the end of the flagellum with no decrease in curvature. However, spermatozoa in which the terminal filament has been removed show a clear 'end effect'. This end effect involves a rapid unbending of bends that have reached the distal end of the flagellum. Computer simulations of flagellar models lacking a terminal filament show a similar end effect. Addition of a terminal filament to the end of the computer model can eliminate the end effect. Realistic bending behaviour of the model is obtained by using a terminal filament with a tapered elastic bending resistance in the basal portion of the terminal filament and a value of $0.03 \times 10^{\circ}$ $\mathrm{pN} \mathrm{nm}{ }^{2}$ in the remainder of the terminal filament. This leads to estimates of $0.01 \times 10^{\circ} \mathrm{pN} \mathrm{nm}^{2}$ for the elastic bending resistance of an individual microtubule, and $0.2 \times 10^{\circ} \mathrm{pN} \mathrm{nm}^{2}$ for the elastic bending resistance of the $9+2$ region of the flagellum. An improvement in propulsive effectiveness by addition of a terminal filament remains to be demonstrated.
\end{abstract}

\section{INTRODUCTION}

A thin terminal filament, about $5 \mu \mathrm{m}$ in length, was noted by Gray (1955) at the end of the sea-urchin sperm flagellum. Its appearance during movement has been recorded photographically in several sperm species under conditions of optimal dark-field or phase-contrast illumination (Brokaw, 1965; Goldstein, 1977; Hiramoto \& Baba, 1978). Various terms, such as terminal piece, end piece, or whiplash, have been used to refer to this structure, which is found in a variety of flagella. We will follow Gray's usage of the term, terminal filament, and will refer to the remaining, major portion of the flagellum, sometimes called the principal piece, as the $9+2$ region of the flagellum.

When examined by electron microscopy, the terminal filament has been found to contain only a subset of the $9+2$ complement of microtubules that is characteristic of the sperm axoneme. A function for the terminal filament has not been established, although it has been suggested that the presence or absence of the terminal filament has a significant effect on the shape of bends near the end of the flagellum (Brokaw, r 965 ) and on the balance of moments near the end of the flagellum (Rikmenspoel, 
1978 ), and may significantly influence the viscous bending moments experienced by the flagellum (Hiramoto \& Baba, 1978).

Previous observations on the terminal filament have been incidental to a description of the structure or behaviour of the $9+2$ region of the flagellum. We describe here new observations on the ultrastructure of the terminal piece, based on a more extensive examination than has been published previously. We have used spermatozoa from the tunicate, Ciona intestinalis and the sea urchin, Lytechinus pictus. These spermatozoa have long flagella and make very planar bending waves, and we have exploited these features to obtain high-resolution photographs showing the bending behaviour of the terminal filament and the bending behaviour of the $9+2$ region of the flagellum in the presence and absence of the terminal filament.

\section{MATERIALS AND METHODS}

C. intestinalis was collected from floating docks in Newport Bay, California, and maintained in circulating sea-water aquaria under constant illumination at the Kerckhoff Marine Laboratory. Animals were dissected to expose and open the sperm duct, and concentrated spermatozoa were collected and stored for short periods on ice at o ${ }^{\circ} \mathrm{C}$.

L. pictus from Mission Bay, California, was maintained in circulating sea-water aquaria at r $3-15{ }^{\circ} \mathrm{C}$. Animals were injected with $0.6 \mathrm{M}$ - $\mathrm{KCl}$ to induce spawning. Concentrated spermatozoa were collected and stored at $0^{\circ} \mathrm{C}$.

\section{Electron microscopy}

Ciona spermatozoa were diluted with an activation solution containing $10 \%$ filtered seawater, $90 \% 0.5 \mathrm{M}-\mathrm{NaCl}$, $10 \mathrm{mM}$-theophylline and $5 \mathrm{mM}$-Tris base (pH 8.1) (Brokaw, 1982c). Demembranation and reactivation were performed with solutions similar to those described by Brokaw (I982c). Lytechinus spermatozoa were diluted into filtered sea-water containing I \% polyvinylpyrolidinone and $\mathrm{o} \cdot \mathrm{I}$ mM-EDTA $(\mathrm{pH} 8 \cdot \mathrm{I})$. Demembranation and reactivation were performed with solutions similar to those described by Brokaw (1979).

To obtain platinum replica preparations, either intact spermatozoa in activation solution or demembranated spermatozoa in reactivation solution were placed on coverslips coated with Alcian Blue (Sommer, 1977). They were then fixed with $2 \%$ glutaraldehyde in $50 \mathrm{~mm}$ phosphate buffer $(\mathrm{pH} 8.0)$. After dehydration through an ethanol series and critical-point drying, they were coated with platinum (approx. $6 \mathrm{~nm}$ thickness) and carbon on a Balzers $360 \mathrm{M}$ freeze-fracture machine. The replicas were removed from the glass coverslips with approximately $\mathrm{O}$ I M-hydrofluoric acid, digested with Chlorox, washed, and picked up on Formvar-coated grids.

For negative-stain preparations, spermatozoa were diluted with $10 \%$ filtered sea-water and $90 \% 0.5 \mathrm{M}-\mathrm{NaCl}$, allowed to stick to Formvar-coated grids, and stained with $0.5 \%$ aqueous uranyl acetate.

The preparations were viewed and photographed using a Philips 20r electron microscope.

\section{Photomicrography of motile spermatozoa}

Photographs showing the movement of the terminal piece were obtained with sperm activated by dilution into sea-water containing $1 \%$ polyvinylpyrolidinone (PVP) and $0.5 \mathrm{~mm}$-EDTA $(\mathrm{pH} 8 \cdot 1)$. Photomicrography was carried out in a room maintained at $18{ }^{\circ} \mathrm{C}$. Spermatozoa were photographed while swimming close to the bottom of a coverglass, using a Zeiss $100 \times$ apochromat objective and ultradarkfield condenser. Illumination was provided by a Varian VIX I $50 F$ Xenon arc lamp, powered in pulsed mode by a Chadwick-Helmuth model ${ }_{1} 36$ power supply, at a flash rate of $200 \mathrm{~Hz}$. A Grass Instruments model $\mathrm{C}_{4} \mathrm{R}$ oscilloscope camera was used for photography on continuously moving $35 \mathrm{~mm}$ film, at a film speed of $0.5 \mathrm{~m} \mathrm{~s}^{-1}$. Kodak 
Tri-X film was used, with a magnification on film of approximately $250 \times$. The film was developed for ASA 6400 in Perfection XR-1 developer.

Films selected for detailed analysis were projected onto the screen of a microfilm reader to give a final magnification of $5700 \times . X, Y$ co-ordinates of points on the centre line of a flagellar image were obtained using a Numonics Corporation model 1220 digitizer in trace mode and transmitted to a Hewlett-Packard HP8 5 microcomputer. In this mode, the digitizer transmits a stream of points as the cursor is moved along the image. The computer checked each incoming point and rejected it if it was separated from the last recorded point by less than a distance equivalent to $0.1 \mu \mathrm{m}$. Usually, about 200 points were recorded along the length of a flagellum about $50 \mu \mathrm{m}$ in length. After tracing an image once, the points were analysed by the following procedures. Every fourth point in the set of points was used to form a subset of points. The angle and distance between each pair of points in the subset was computed, and this information was then converted to give angular orientation of the flagellum as a function of flagellar length at uniform intervals (corresponding to $0.4 \mu \mathrm{m}$ ) along the length. The resulting stepwise curve was then smoothed by two passes of a smoothing process that replaced each angle, $A(i)$, with $0.25 A(i-1)+0.5 A(i)+0.25 A(i+\mathrm{I})$. This process was then repeated on each of the other three subsets of points that could be obtained from the original set of points by taking every fourth point, with different starting points, and the results from the four subsets were averaged together. This procedure is similar to that used by Johnston, Silvester $\&$ Holwill (1979) and Johnston (1978) to analyse streams of points from an automatic curvetracker; and by Hiramoto \& Baba (1978) and Brokaw, Luck \& Huang (I982) to average data from repeated tracings of images. Each image tracing averages results from four subsets of points with an average spacing of about $1 \mu \mathrm{m}$, similar to the sets of points obtained from each tracing by Hiramoto \& Baba (1978); however, the subsets are probably not completely independent as errors in tracking the centreline of the flagellar image may be correlated.

The procedure described above was repeated twice more for each image, and the three completely independent tracings were stored on disk, and subsequently averaged to produce the curves in Figs. 5-7, 9. For each tracing, the origin of the flagellum was relocated relative to the digitizer grid, to avoid systematic errors. The image of the sperm head usually has a bright periphery and a thin, elongated dark area; these features were used as a convenient marker for the orientation of the sperm head, and all angles were referenced to a line through this elongated dark area. Some of the sources of errors in these procedures are discussed by Brokaw et al. (1982).

\section{Computer simulations}

Techniques for computer simulation of flagellar movement have been described in a series of earlier papers. These techniques solve numerically a partial differential equation that balances active, viscous and elastic bending moments at points along the length of the flagellum (Brokaw, 1972 , $1982 b$; Hines $\&$ Blum, r 978 ). The solution of this equation, as a function of time, represents the motion of a flagellar model defined by the partial differential equation. The flagellar model must contain a prescription for the active moments that are responsible for bending of a flagellum. Successful models that show spontaneous oscillation and bend propagation were first obtained by including a simple mathematical relationship expressing the control of active shear moment by curvature (Brokaw, 1972; Hines \& Blum, 1978). In subsequent development of these models, this prescription has been replaced by explicit incorporation of the kinetics and mechanics of the dynein cross-bridges that produce active shear moment, and statements about the control of cross-bridge kinetics by flagellar curvature (Brokaw \& Rintala, 1975; Hines \& Blum, 1979; Blum \& Hines, 1979; Brokaw, 1980, 1982a, $b$ ). Since the present work focuses on the movement of the tarminal filament, which appears to have no internal mechanism for generating active shear, the details of the mechanism for generating and controlling active shear moment are irrelevant and not described here. The computer simulations performed here have, for convenience, used the current model, which is similar to those described by Brokaw $(1982 a, b)$.

In modelling the behaviour of the terminal filament, two aspects of the hydrodynamic theory need attention. One is the effect of the reduced diameter of the terminal filament. The computer simulation procedures use the drag coefficient method of Gray \& Hancock (I955) 
(resistive force theory) to evaluate the viscous drag forces acting on each segment of the flagellar model. The drag coefficients used were determined by more recent work (Lighthill, 1976; Johnson \& Brokaw, 1979). The normal and tangential drag coefficients are given, respectively, by:

$$
C_{N}=\frac{4 \pi \eta}{\ln (0 \cdot 18 L / b)+\mathrm{I} / 2} \text { and } C_{L}=\frac{2 \pi \eta}{\ln (0 \cdot 18 L / b)}
$$

where $\eta=$ viscosity, $L=$ wavelength measured along the flagellum, and $b=$ the radius of the filament. With $L=30 \mu \mathrm{m}$ and $b=0.1 \mu \mathrm{m}$ (for the $9+2$ portion of the flagellum), $C_{L} / \eta=$ $\mathrm{I} \cdot 58$ and $C_{N} / C_{L}=\mathrm{I} \cdot 77$. With $L=30 \mu \mathrm{m}$ and $b=0.04 \mu \mathrm{m}$ (for the terminal filament), $C_{L} / \eta=\mathrm{I} \cdot 21$ and $C_{N} / C_{L}=\mathrm{I} \cdot 8 \mathrm{1}$. For our computations, the value of $C_{N} / C_{L}=\mathrm{I} \cdot 8$, used in previous simulations, has been retained for both portions of the flagellum, and the value of $C_{L}$ used in the terminal filament is $80 \%$ of the value used normally for the $9+2$ portion of the axoneme. These expressions indicate that the values of the drag coefficients are not very sensitive to the diameter of the flagellum, since a reduction in diameter by $60 \%$ results in a reduction of only about $20 \%$ in the values of the drag coefficients. We do not therefore need to obtain an exact value for the diameter of the terminal filament.

The second consideration is a hydrodynamic 'end effect'. The expressions given above for $C_{N}$ and $C_{L}$ effectively ignore any effects resulting from the ends of the filament, and give the drag per unit length for a small segment immersed in a filament that is long compared to the wavelength, $L$. Actually, the region near the tip of a moving filament will experience increased viscous drag, because it will be moving through a region of fluid that is not being propelled in the same direction by more distal regions of the filament, as would be the case if the region were within a long filament rather than at the tip. An estimate of the magnitude of this effect can be obtained by using equation (18) from Keller \& Rubinow (1976), which expresses the force distribution along the length of a uniform cylinder moving in a direction at right angles to its axis. For instance, for a cylinder with a radius of $0.04 \mu \mathrm{m}$ and a length of $10 \mu \mathrm{m}$, the average force on a I $\mu \mathrm{m}$ segment at the end of the cylinder is $18 \%$ greater than the average force per $1 \mu \mathrm{m}$ for the entire cylinder. This percentage increases with the length of the cylinder, to $32 \%$ if the length is $100 \mu \mathrm{m}$. This calculation suggests that the hydrodynamic effect at the end of a flagellum is unlikely to amount to more than a $20-30 \%$ increase in viscous drag on the terminal $\mathrm{I} \mu \mathrm{m}$ of the terminal filament. A $25 \%$ increase for the last I $\mu \mathrm{m}$ segment has been used in the computations reported here.

The computations reported here were performed with a flagellar model having a length of $45 \mu \mathrm{m}$ for the $9+2$ region of the flagellum, and the model was divided into segments $\mathrm{I} \mu \mathrm{m}$ in length for the purposes of computation. The model is slightly shorter than the real flagellum, which conserves somewhat on computational time and cost. A total of 800 time steps was computed for each cycle of oscillation of the model. Computations were routinely carried out for six cycles of oscillation, in order to allow the movement to stabilize. For the $9+2$ region of the flagellum, the value of elastic bending resistance, $E_{B}$, was $0.2 \times 10^{\circ} \mathrm{pN} \mathrm{nm}^{2}$, and the elastic shear resistance, $E_{S}$, was of the non-linear form suggested by Hines \& Blum (I978) with a constant $A_{1}=36 \mathrm{pN}$. The value of $C_{L}$ used for this portion is $2.13 \times 10^{-9} \mathrm{pN} \mathrm{s} \mathrm{nm}^{-2}$.

\section{RESULTS}

\section{Structure of the terminal filament}

Light micrographs of Ciona spermatozoa, such as Fig. 4A, indicate that the major portion of the flagellum, which we will refer to as the $9+2$ region or the $9+2$ axonemal region, has a uniform diameter and a length of $48-49 \mu \mathrm{m}$. The image of the terminal filament is much thinner, with a length of $5-6 \mu \mathrm{m}$. In the best photographs of Ciona spermatozoa, there appears to be a distinct transition between the $9+2$ region and the terminal filament, but there is also some further reduction in image intensity along the length of the terminal filament. 

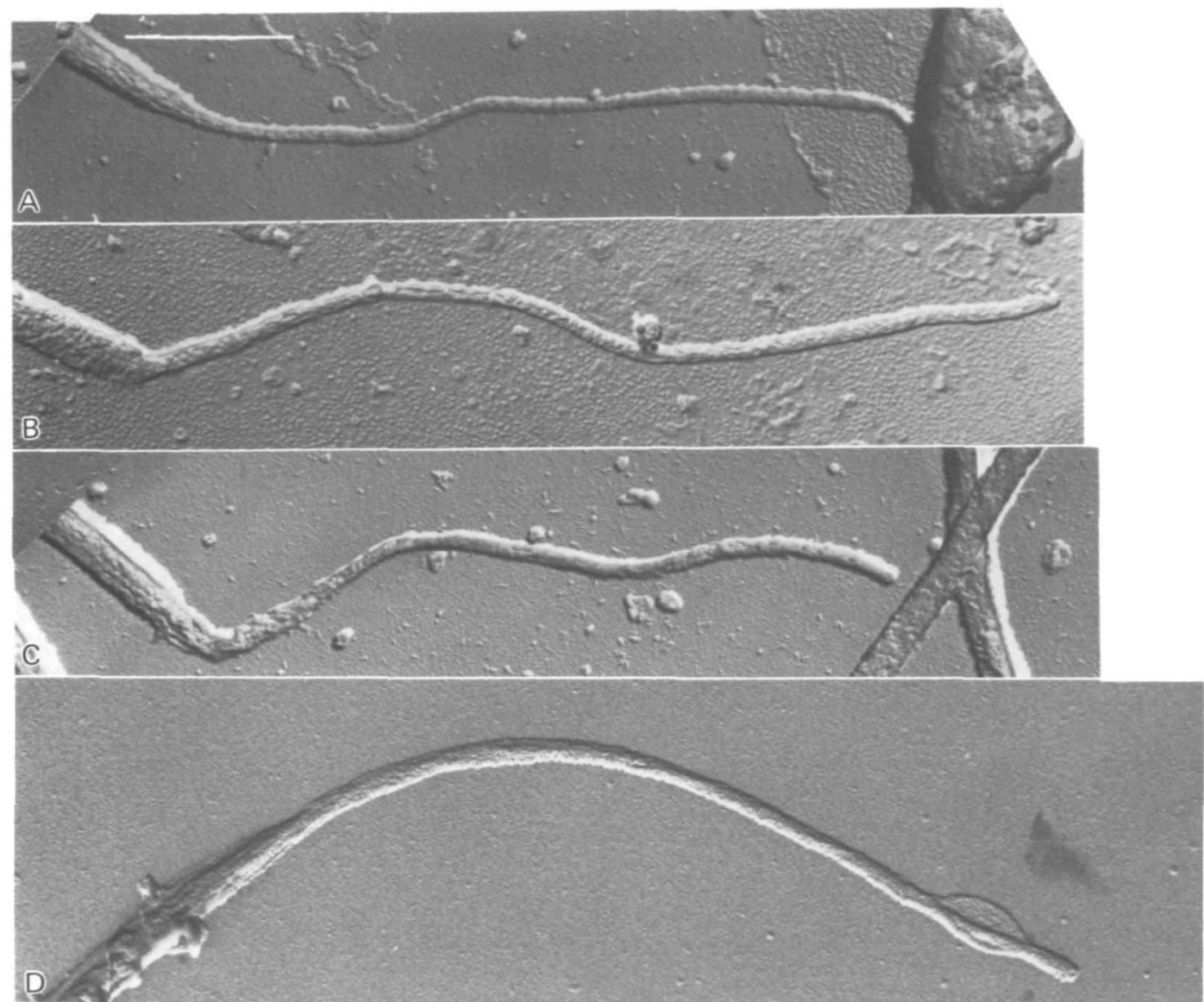

8

$E$

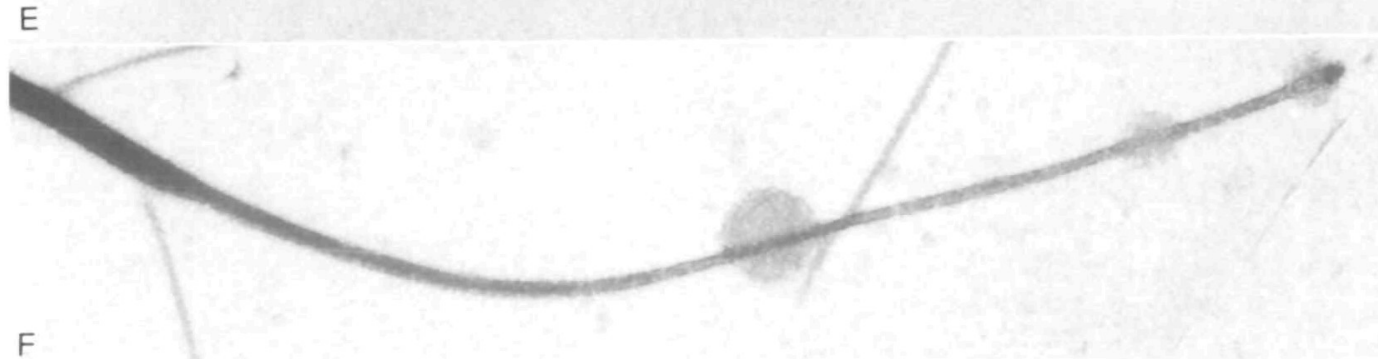

Fig. 1. Electron micrographs of intact terminal filaments at the ends of Ciona (A-E) and Lytechinus (F) sperm flagella. A to D are platinum replicas; E and F are negatively stained. Bar (A), I $\mu \mathrm{m}$. 


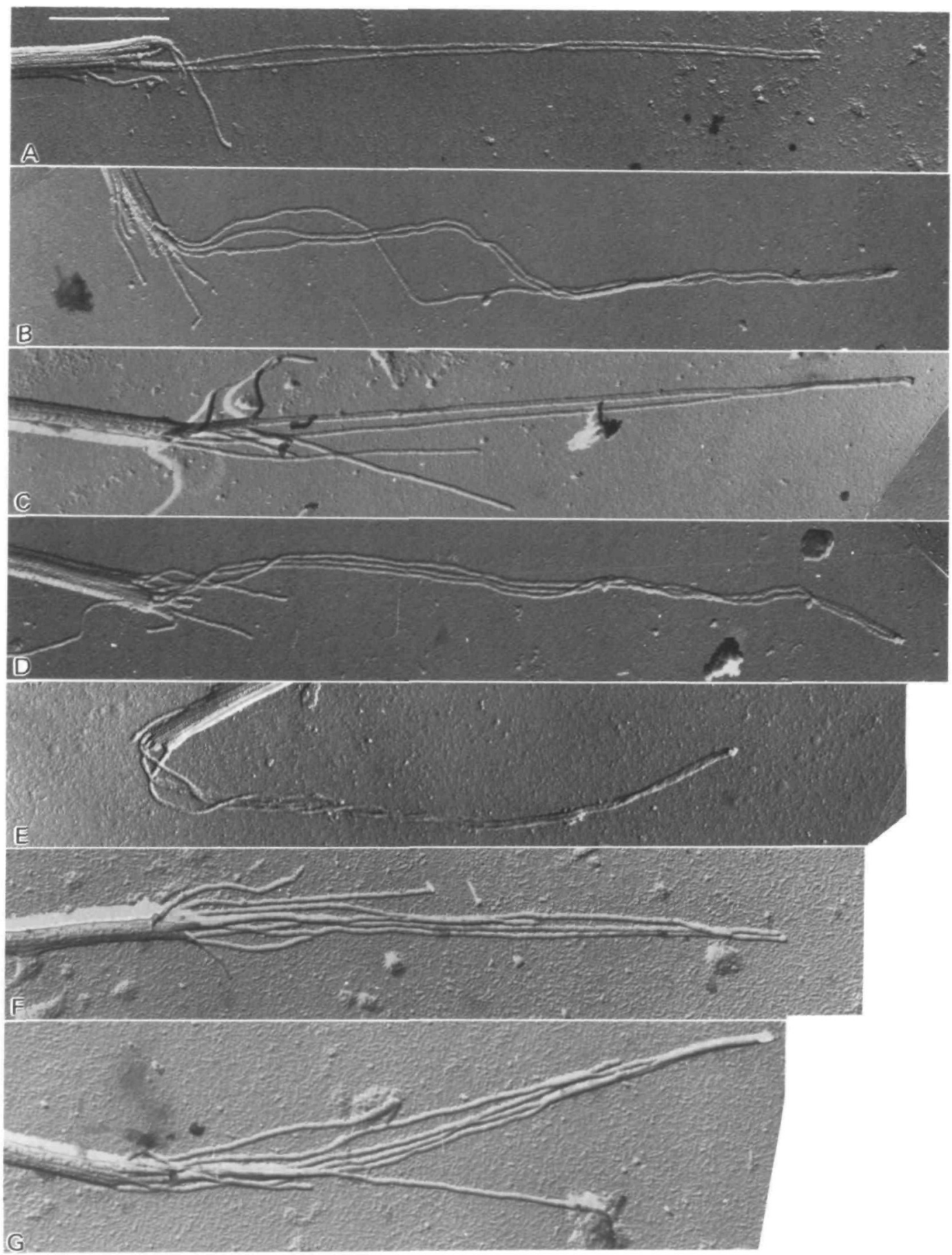

Fig. 2. Electron micrographs of platinum replicas of demembranated terminal filaments. A, B, D, E, Ciona; C, F, G, Lytechinus. C, F, G appear thicker due to change in shadow angle. Bar $(A), 1 \mu \mathrm{m}$. 
Electron micrographs of platinum replicas of intact flagella, shown in Fig. IA-D, appear to present the least-damaged picture of the terminal filament. These micrographs indicate the presence of three distinct regions near the distal end of the Ciona flagellum:

(I) A region of uniform width (about $0.24 \mu \mathrm{m}$ ) corresponding to the $9+2$ axonemal region that makes up the major portion of the length of the flagellum. This is shown on the left-hand side of Fig. I A-D.

(2) A region of almost uniform width $(0.08-0.10 \mu \mathrm{m})$ that makes up most of the length of the terminal piece. In the examples shown in Fig. I this region has a length of $4-5 \mu \mathrm{m}$.

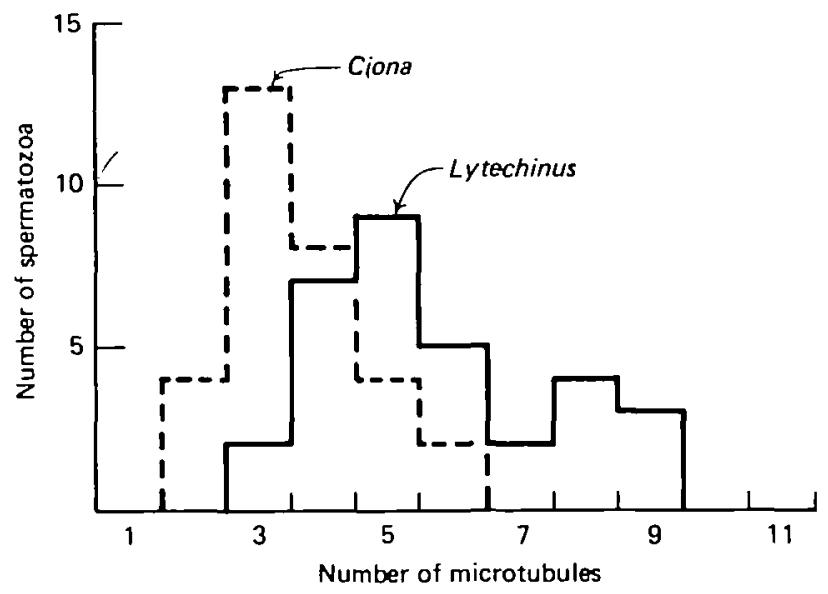

Fig. 3. Number of microtubules found $1 \mu \mathrm{m}$ from the end of the $9+2$ region of sperm flagella using platinum replicas of demembranated Ciona and Lytechinus spermatozoa.

(3) A transition region between regions (I) and (2), which may be up to $\mathrm{I} \mu \mathrm{m}$ in length. In this region, there is either a gradual tapering between the widths of the two longer regions, or a relatively abrupt decrease in width at the end of region ( 1 ), followed by a region that is slightly wider than region (2) and shows a very slight taper.

Fig. IE, F shows intact terminal filaments, but in this case negative staining has been used, which allows internal structure to be seen within the intact membrane. Fig. I 2 suggests that region (2) of Ciona contains one to three microtubular elements. Since the membrane appears to be intact, this small number of microtubular elements is probably not the result of losses during the preparation of the specimens for electron microscopy. In Lytechinus (Fig. I F), we cannot make a confident count of microtubules.

Electron micrographs of platinum replicas of demembranated flagella are shown in Fig. 2. In these preparations, considerable damage to the terminal filaments was found, and the apparently least-damaged examples were selected for Fig. 2. These micrographs indicate that region (2), the major portion of the Ciona terminal filament, consists of two to four microtubules, in reasonable agreement with the negatively stained examples of intact terminal filaments in Fig. I E. However, Lytechinus flagella 

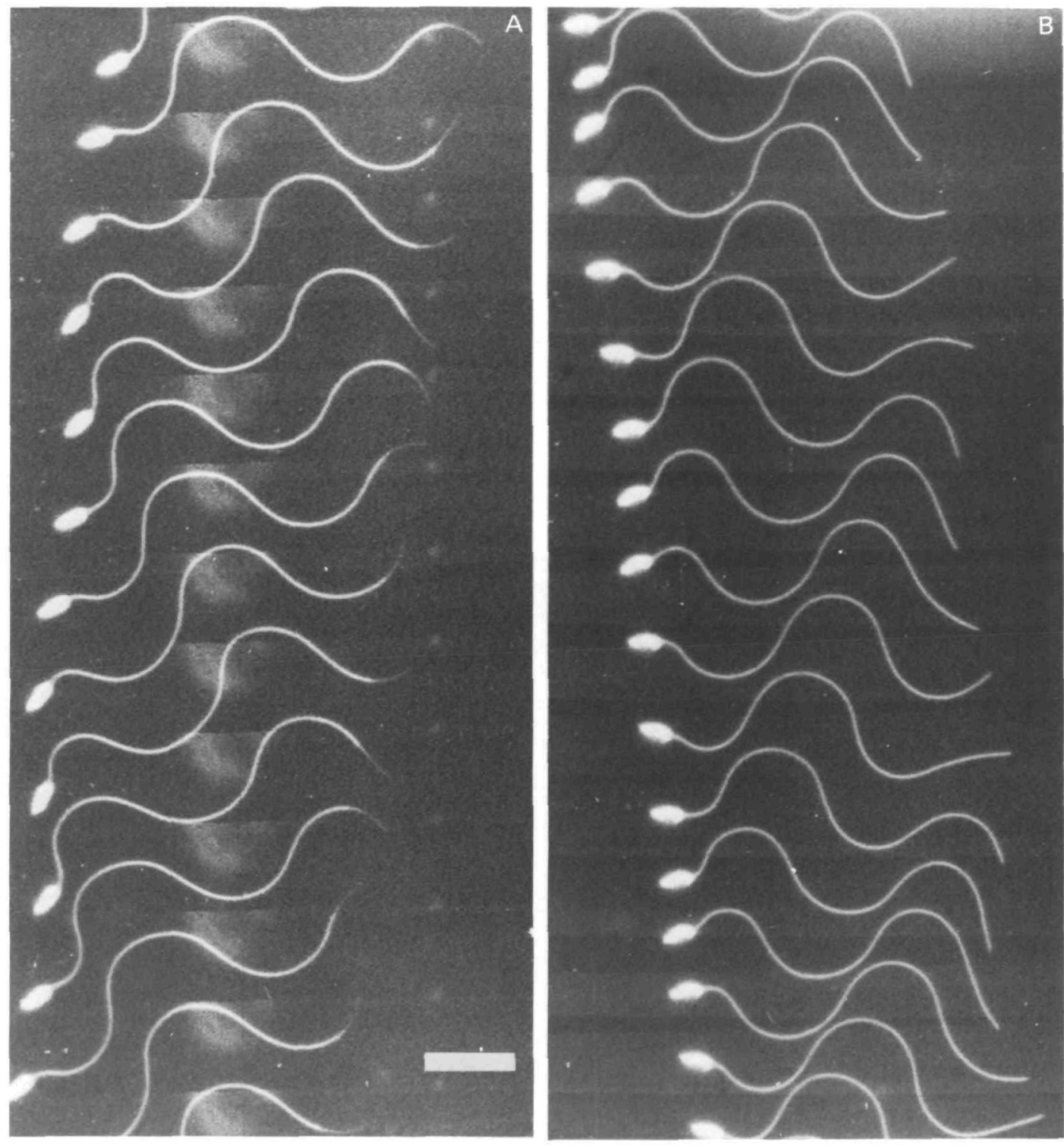

Fig. 4. Multiple-flash photographs on moving film showing Ciona spermatozoa swimming in sea-water. In A, a terminal filament is present at the end of the flagellum, and the sequence of images is from top to bottom. In $\mathrm{B}$, the terminal filament has been removed from the flagellum, and the sequence of images is from bottom to top. The flash rate is $200 \mathrm{~Hz}$. Bar, $10 \mu \mathrm{m}$.

have more and longer $A$ tubules extending into the terminal filament. This difference between Ciona and Lytechinus can be demonstrated by a count of microtubules at a fixed distance distal to the termination of the $9+2$ region (Fig. 3). At I $\mu \mathrm{m}$ distance, Lytechinus flagella have a broad distribution of microtubule number, ranging from three to nine, with over $70 \%$ having five or more microtubules. At the same distance, Ciona flagella have two to six microtubules with $80 \%$ having four or fewer microtubules. 
The two central-pair microtubules are identifiable in these micrographs by their connection together near the $9+2$ region (Fig. $2 \mathrm{~A}-\mathrm{C}, \mathrm{F}, \mathrm{G}$ ) and/or by the presence of the cap-like structure that joins them together at the distal tip of the terminal filament (Fig. 2B-E). The central pair is present through the entire length of the terminal filament in all seven of the examples shown in Fig. 2.
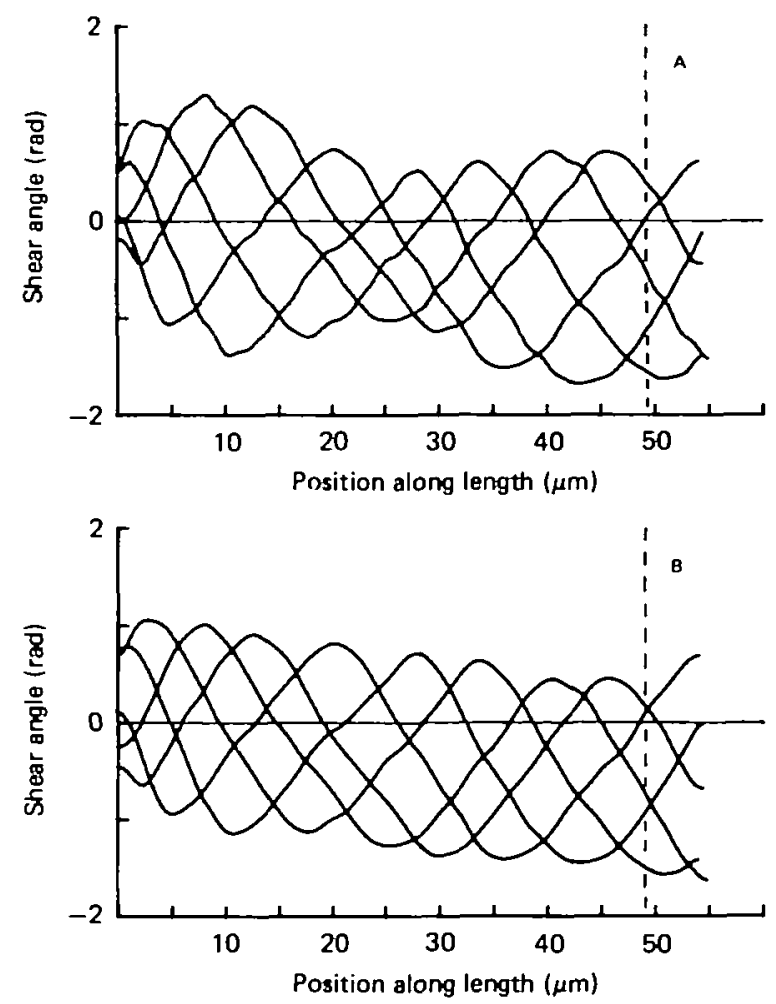

Fig. 5. Shear curves showing angular orientation as a function of length along the flagellum, obtained from images 6 to 10 of Fig. 4A. The curves in B have been subjected to four additional cycles of smoothing (see Materials and Methods) and repositioned vertically to eliminate the apparent oscillatory synchronous sliding. The beat frequency of this flagellum is slightly greater than $4 \circ \mathrm{Hz}$. The broken line indictaes the approximate position of the junction between the $9+2$ region of the flagellum and the terminal filament.

\section{Bending behaviour of the distal end of the flagellum}

Fig. $4 \mathrm{~A}$ is our best photograph showing the entire length of the Ciona sperm flagellum, including the terminal filament, in sharp focus, with maximum contrast. Other photographs were obtained in which this amount of detail could be seen easily in the projected negatives, but which did not produce as satisfactory prints for reproduction. We measured the depth of focus of the microscope optics used for these photographs, by photographing the terminal filament of a dead spermatozoon at various settings of the calibrated fine-focus adjustment of the mic oscope. This procedure indicated that the range of sharp focus was approximately $\mathrm{I} \mu \mathrm{m}$ in depth. 

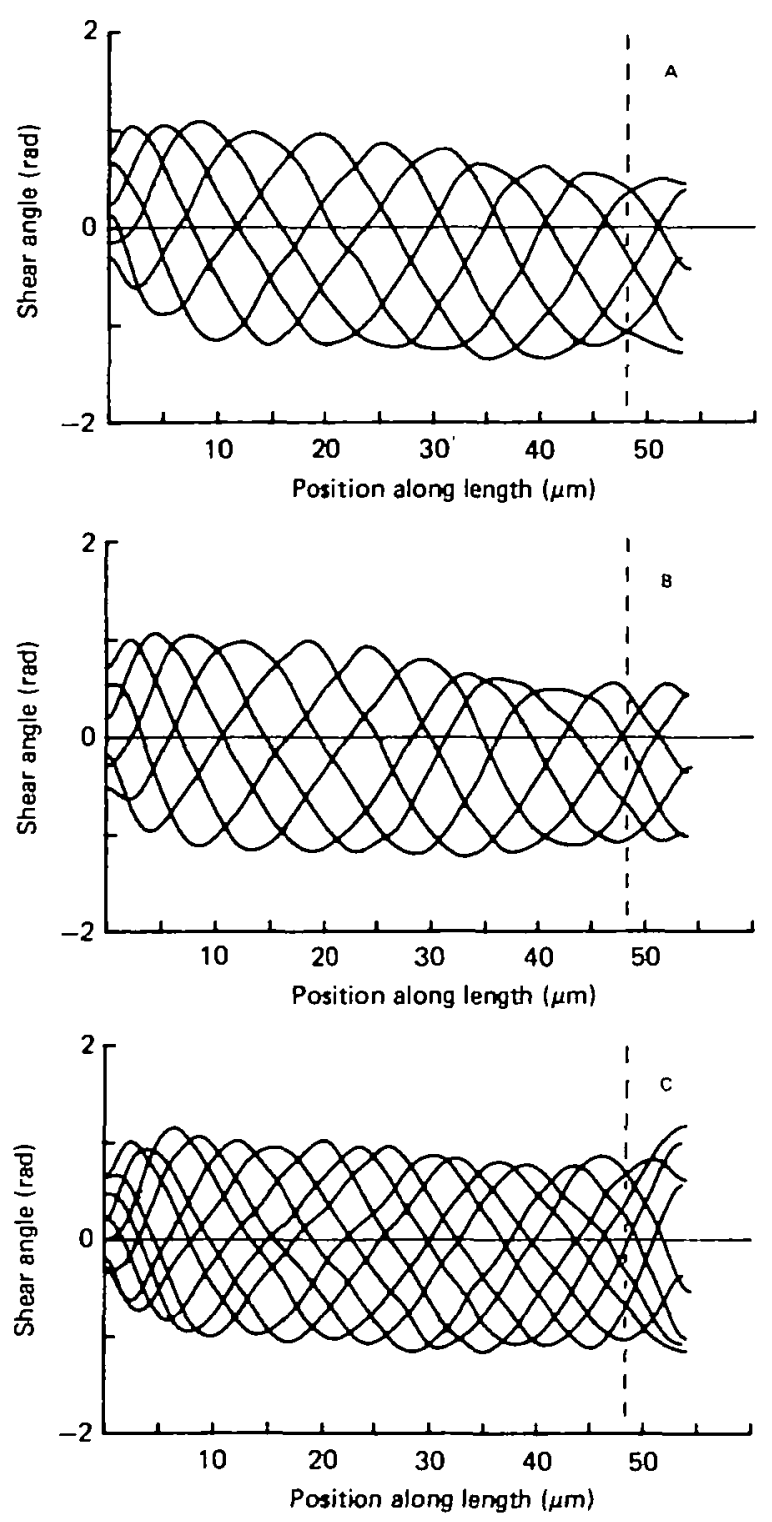

Fig. 6. Shear curves, smoothed and repositioned as in Fig. $5 \mathrm{~B}$, for three additional examples of flagella with intact terminal filaments. In $\mathrm{A}$, there are six images evenly spaced throughout one beat cycle, so the beat frequency is $33-34 \mathrm{~Hz}$. In $\mathrm{B}$, the beat frequency is slightly higher. In $\mathrm{c}$, there are nine images covering two beat cycles; the beat frequency is approximately $45 \mathrm{~Hz}$. The broken line indicates the approximate position of the junction between the $9+2$ region of the flagellum and the terminal filament. 
Since even the images of the terminal filament that appear straight in Fig. $4^{\mathrm{A}}$, such as images 2 and 9 from the top of Fig. $4 \mathrm{~A}$, appear to be in sharp focus, we can eliminate the possibility that the changing shape of the image of the terminal filament is the result of rotation of a curved terminal filament. This possibility was suggested by observations on other flagella in which the central pair of microtubules extends beyond the $9+2$ region of the flagellum and has been shown to rotate (Jarosch \& Fuchs, 1975; Omoto \& Witman, 1981; Kamiya, Nagai \& Nakamura, 1982).

Fig. 5A shows the results of tracing images 6 to ro on this photograph to obtain curves of angle versus position along the flagellum. As in previous work (Brokaw, 1979, etc.) these curves are interpreted as shear curves that provide an angular measure of the amount of shear between axonemal doublet microtubules. This interpretation depends on the assumptions that there is no shear between tubules at the basal end of the flagellum, that the axoneme does not twist, and that the microtubular doublets are inextensible. The shear curves in Fig. $5 \mathrm{~A}$ show a constriction of the envelope of the curves in the mid-region of the flagellum that has been attributed to the presence of oscillatory 'synchronous sliding' between flagellar doublet microtubules (Brokaw, 1979; Gibbons, 198I). However, this interpretation depends on the assumption that the orientation of the basal end of the flagellum remains constant relative to the axis of the sperm head, and this assumption has been questioned (Gibbons, I98I). In Fig. 5 $\mathrm{B}$, the same shear curves have been subjected to four additional cycles of smoothing, as described in Materials and Methods, and repositioned to eliminate the apparent oscillatory synchronous sliding. This repositioning would be valid if the apparent oscillatory synchronous sliding is an artifact resulting from error in the assumption that the head axis is a valid indicator of the angular orientation of the basal end of the flagellum. The validity of this procedure has not been established, but it is used here and in Figs. 6, 7 and 9 to simplify the shear pattern so that the behaviour of the distal end of the flagellum can be seen more clearly.

Shear curves from three additional photographic sequences that gave clear images of the movement of the terminal filament are shown in Fig. 6.

After shearing the sperm suspension by forcing it in and out of a Pasteur pipette, we were able to find spermatozoa where the terminal filament was lacking, but with the $9+2$ region of the flagellum retaining all or nearly all of its normal length. A photograph of one of these spermatozoa is shown in Fig. 4B. Shear curves for this flagellum are shown in Fig. $7 \mathrm{~A}$, and for two other examples of flagella lacking the terminal filament in Fig. 7B, C. Some photographs of spermatozoa in these preparations appeared to show only the short transition region at the end of the $9+2$ region of the flagellum, with the major portion of the terminal filament missing. These flagella showed bending behaviour indistinguishable from that of flagella completely lacking the terminal filament (data not shown).

Examination of the photographs and shear curves for sperm flagella with and without a terminal filament leads to the following three conclusions: ( 1 ) when the terminal filament is present, bends are propagated smoothly off the end of the flagellum, with no obvious discontinuity in bending behaviour at the transition between the $9+2$ region and the terminal filament. (2) When the terminal filament is absent, bends at 

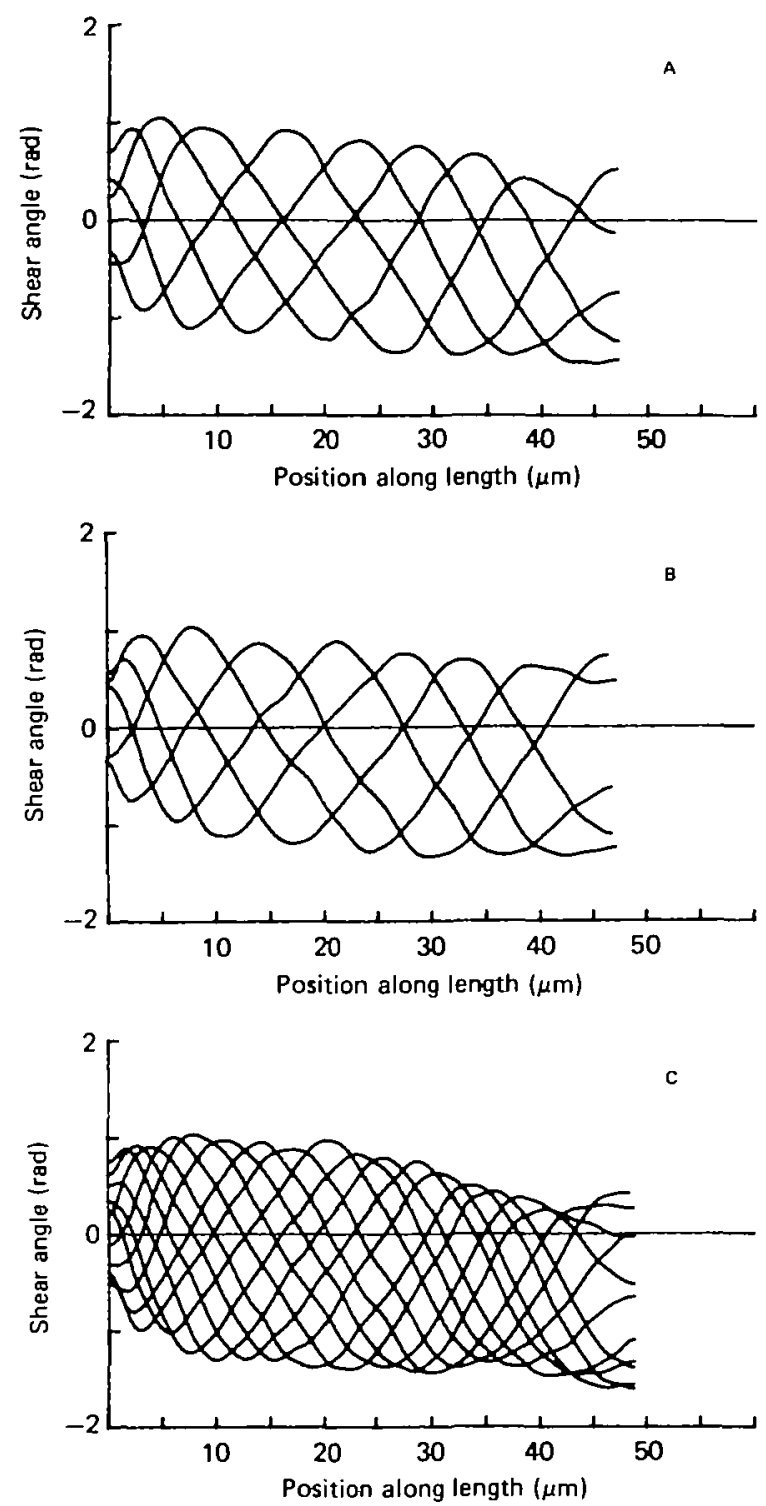

Fig. 7. Shear curves, smoothed and repositioned as in Fig. $5 \mathrm{~B}$, for three examples of flagella without terminal filaments. In $A$ and $B$, there are five images that almost cover one beat cycle; the beat frequency is $38-39 \mathrm{~Hz}$. In c, there are I I images covering two beat cycles; the beat frequency is about $36 \mathrm{~Hz}$.

Fig. 8. Multiple-flash photographs on moving film showing Lytechinus spermatozoa attached to the coverglass by their heads. In $\mathrm{A}$, a terminal filament is present at the end of the flagellum. In $\mathbf{B}$, the terminal filament has been removed from the flagellum. In both, the sequence of images is from top to bottom. Flash rate is $200 \mathrm{~Hz}$. Bar, $10 \mu \mathrm{m}$. 

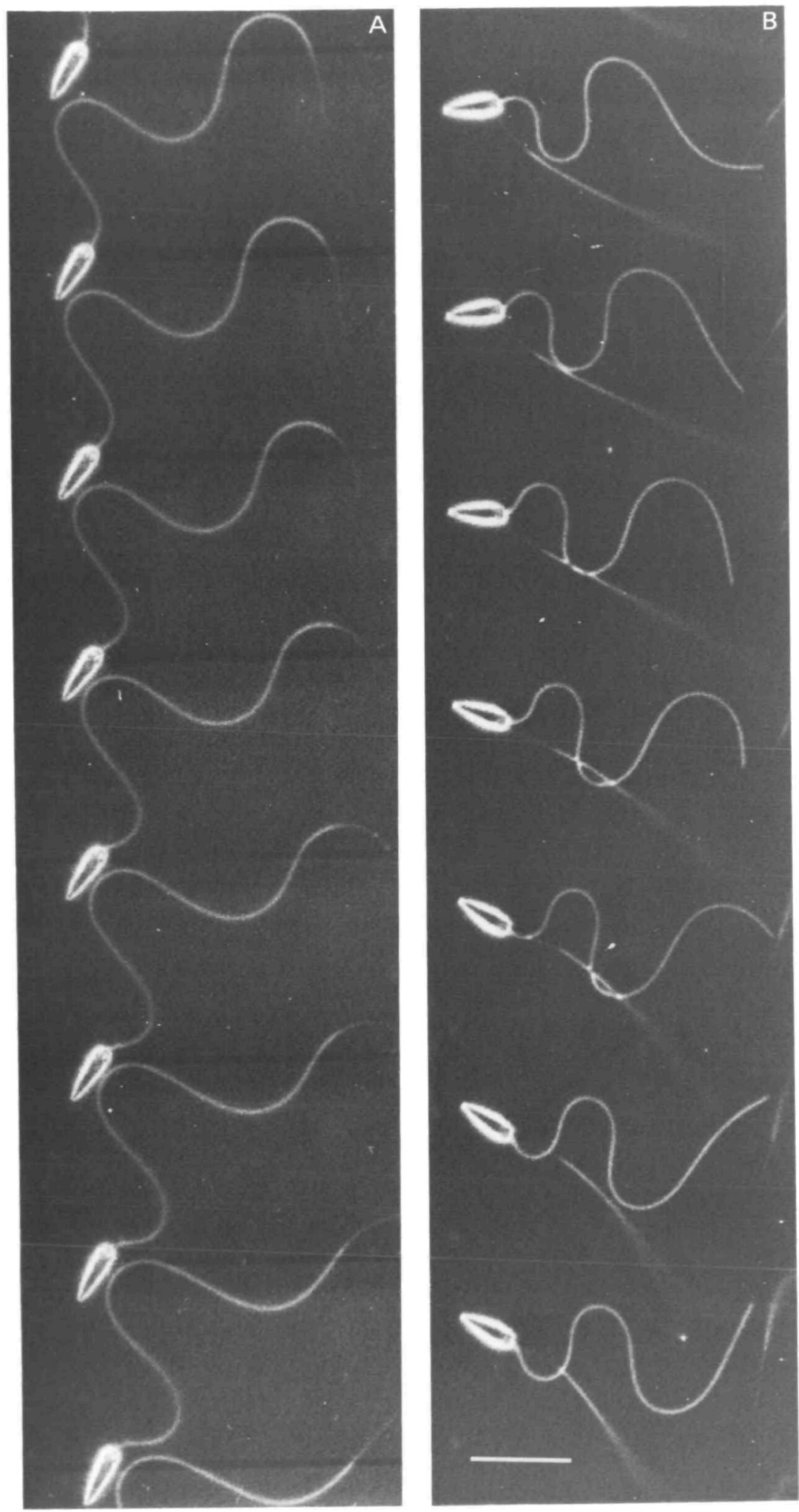
the end of the flagellum decrease rapidly in curvature, causing an obvious end effect. The presence of the terminal filament thus modifies the behaviour of the distal end of the $9+2$ region of the flagellum, so that this end effect is eliminated from the bending behaviour of the $9+2$ region of the flagellum. (3) The bending behaviour of the terminal filament does not show an end effect similar to that seen when the flagellum is terminated in the $9+2$ region. Instead, bends extending into the terminal filament retain a nearlv normal curvature until they propagate off the end of the filament. In
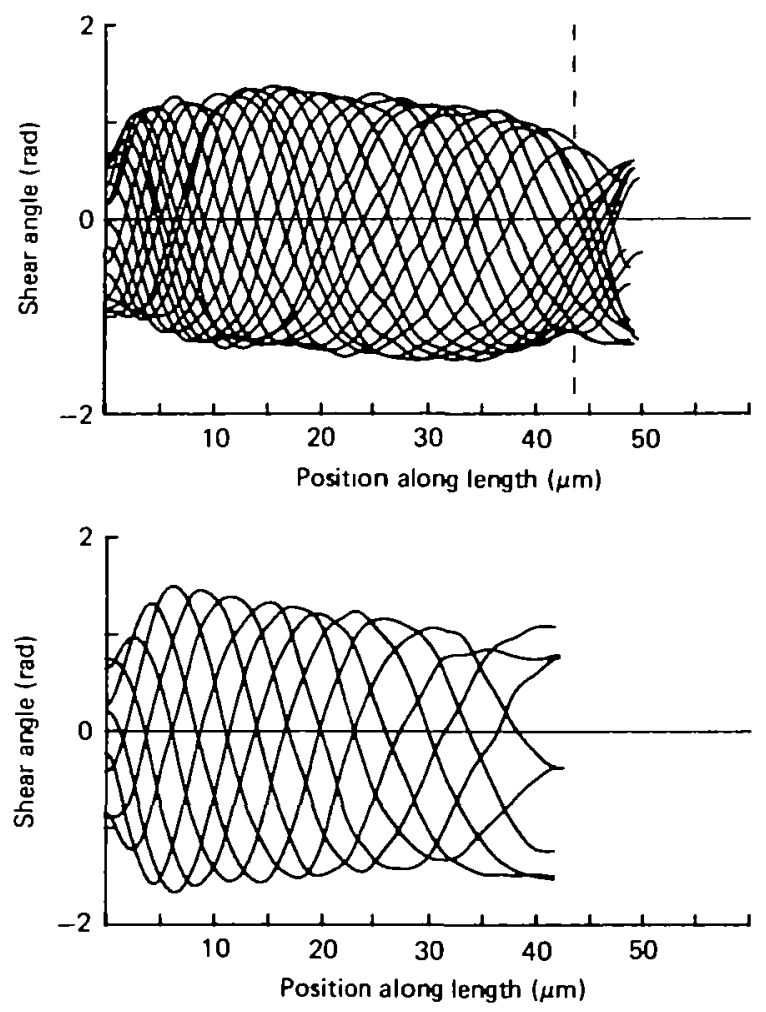

Fig. 9. Shear curves, smoothed and repositioned as in Fig. $5 \mathrm{~B}$, showing angular orientation as a function of length along attached Lytechinus flagella. The curves in $A$ are for a flagellum with intact terminal filament with a beat frequency of approximately II Hz. The broken line indicates the approximate position of the junction between the $9+2$ region of the flagellum and the terminal filament. The curves in $B$ are for a flagellum without a terminal filament beating at approximately $25 \mathrm{~Hz}$.

some cases, the curvature of bends in the terminal filament is greater than that seen in the $9+2$ region of the flagellum. An example of this is shown by the shear curves in Fig. $6 \mathrm{c}$, where the shear curves have a steeper slope in the distal portion corresponding to the terminal filament. This effect has been seen only in spermatozoa swimming with high beat frequencies (greater than $35 \mathrm{~Hz}$ ).

Lytechinus spermatozoa attached to the slide by their heads were also analysed, since bend angles of attached spermatozoa are larger than free-swimming ones. We picked slowly beating examples here for higher time resolution (Fig. 8). Fig. 9 shows 
shear curves from such sequences. Attached flagella clearly have higher bend angles (Brokaw, 1965). As in free-swimming Ciona flagella, bends are propagated smoothly off the distal end when the terminal filament is present, but there is an obvious end effect in its absence. Furthermore, the bend in the terminal filament reflects the higher bend angle of the attached flagellum. Thus, under quite different conditions we still observe effects of the terminal filament on the movement of sperm flagella.

\section{Computer simulations}

Output from a computer simulation of a flagellar model $45 \mu \mathrm{m}$ in length, with no terminal filament at the distal end, is shown in Fig. 10. In this case, the frequency of oscillation was $4 \circ \mathrm{Hz}$. The movement of the distal end resembles that obtained with
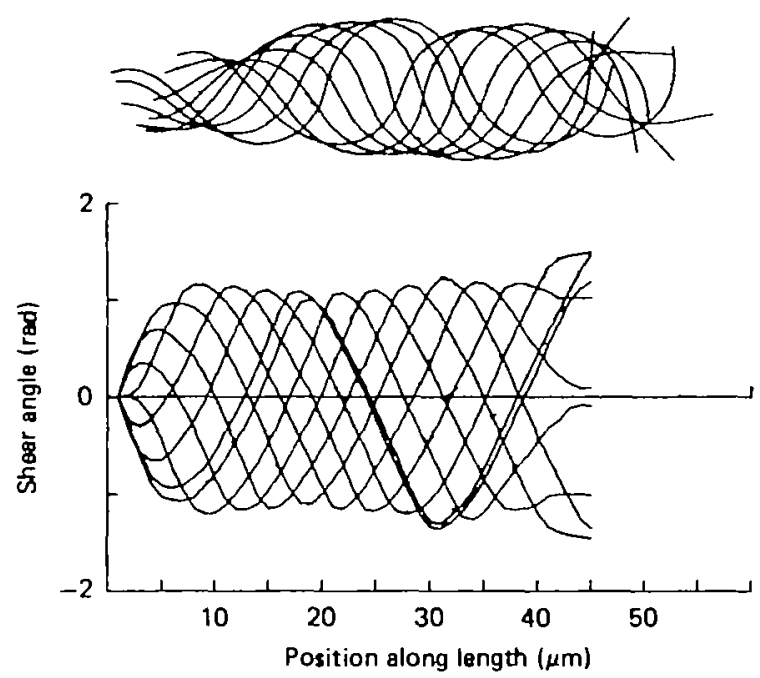

Fig. 10. Output from computer simulation of a model flagellum without a terminal filament. There are nine images covering a period equivalent to $25 \mathrm{~ms}$; the frequency of oscillation of the model is close to $40 \mathrm{~Hz}$.

earlier computer simulations (e.g., see Brokaw, 1972), and shows a distinct end effect, in which bends that have reached the end of the flagellum straighten out, rather than being propagated off the end of the flagellum with constant curvature. There are some differences between the shear curves obtained with the model flagellum (Fig. ro) and the shear curves obtained with real flagella when the terminal filament is absent (Fig. 7): the behaviour at the basal end of the flagellum is different, and the real shear curves indicate that the bending waves are not completely symmetrical. However, at the distal end of the flagellum the characteristics of the shear curves are similar.

Fig. I I shows the output from a computer simulation in which a terminal filament of length $6 \mu \mathrm{m}$ has been added to the distal end, so that the total length is $51 \mu \mathrm{m}$. In this case, the terminal filament is given the following properties: no active moment is generated, the elastic bending resistance, $E_{B}$, is the same as in the $9+2$ region of the flagellum, and there is no significant elastic shear resistance, $E_{S}$. With these proper- 
ties, the addition of the terminal filament modifies the behaviour of the last $5 \mu \mathrm{m}$ of the $9+2$ region of the flagellum, from 40 to $45 \mu \mathrm{m}$, so that the end effect seen in Fig. I I is absent from this region of the flagellum, but it is transferred distally to the terminal filament. This terminal filament therefore accomplishes one of the effects of the terminal filament on real flagella, the removal of the end effect on the $9+2$ region of the flagellum. However, the movement of the terminal filament itself does not match the movement of the terminal filament on real flagella, since it displays a definite end effect.

Fig. 12 shows the output from a computer simulation in which the elastic bending resistance, $E_{B}$, in the terminal filament is reduced to $15 \%$ of the value used for the $9+2$ region of the flagellum. This would be the appropriate value for a terminal
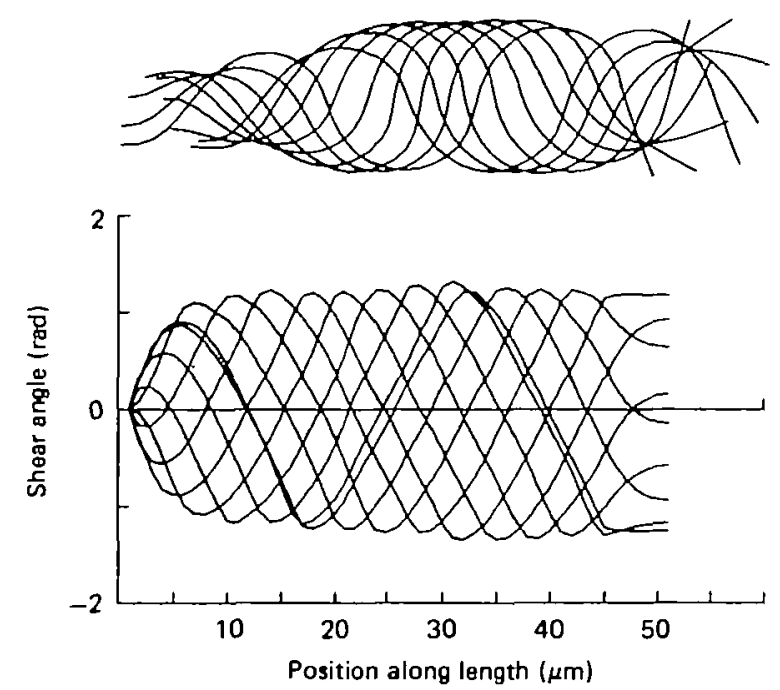

Fig. I I. Output from computer simulation of a model flagellum with a terminal filament having the same stiffness used for the $9+2$ region of the axoneme, $0.20 \times 10^{9}$ $\mathrm{pN} \mathrm{nm}^{2}$. There are nine images covering a period equivalent to $26.67 \mathrm{~ms}$; the frequency of oscillation is slightly less than $37.5 \mathrm{~Hz}$. The junction between the $9+2$ region of the flagellum and the terminal filament is at $45 \mu \mathrm{m}$.

filament containing three microtubules, if the two single microtubules and nine doublet microtubules in the $9+2$ region are considered to have a bending resistance equivalent to 20 single microtubules. The results in this case show a different kind of end effect. In this case, the terminal filament bends excessively. This can be easily seen by examination of both the shear curves and the images of the flagellum. In addition, the end effect in the distal portion of the $9+2$ flagellum is not completely eliminated. The shear curves show a decrease in curvature of bends passing through this region. This terminal filament appears to have a bending resistance too low to resist the viscous bending moment imposed on it by movement through the medium and pass on the effect of this moment to the adjacent $9+2$ region of the flagellum.

Fig. I 3 shows the output from a computer simulation in which $E_{B}$ decreases gradually in the terminal filament. In the computer model, which has a terminal 

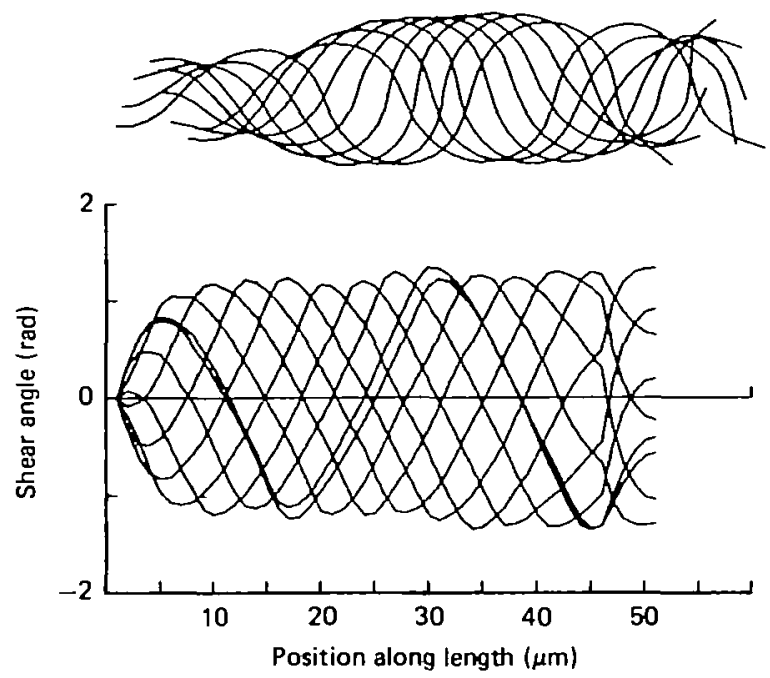

Fig. 12. Output from a computer simulation of a model flagellum with a terminal filament having a reduced stiffness, equal to $0.03 \times 10^{\circ} \mathrm{pN} \mathrm{nm} \mathrm{m}^{2}$. There are nine images covering a period equivalent to $26.67 \mathrm{~ms}$; the frequency of oscillation is slightly less then $37 \cdot 5 \mathrm{~Hz}$.
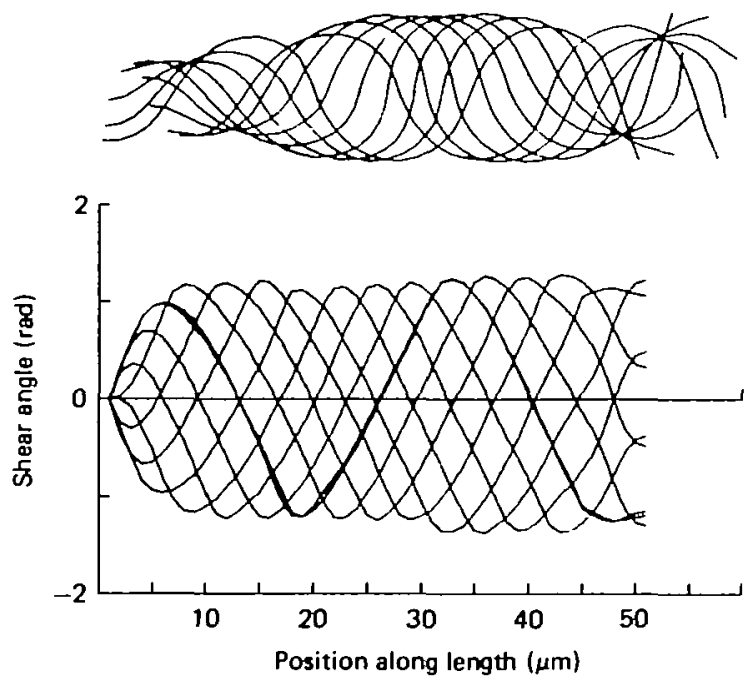

Fig. 13. Output from a computer simulation of a model flagellum with a terminal filament having a tapered stiffness, as described in the text. There are nine images covering a period equivalent to $26.67 \mathrm{~ms}$; the frequency of oscillation is $37.5 \mathrm{~Hz}$.

filament consiting of six I- $\mu \mathrm{m}$ segments, there are five joints between segments in the terminal filament. The values given to $E_{B}$ at these joints, proceeding distally, were $0.5,0.25,0.15,0.15$ and 0.15 times the value of $E_{B}$ used for the $9+2$ region of the flagellum. In this case, the end effect is nearly eliminated, and the distal end of the flagellum shows behaviour very similar to that seen in real flagella with terminal filaments. The shear curves in Fig. I 3 indicate that there is some increase in curvature 
in the terminal filament, but this is also seen in some of the shear curves for the real flagella, such as Fig. 6c. In other computations (not shown) we were able to obtain results in which the end effects were eliminated and the curvature was constant throughout the terminal bend region. This required a more tapered elastic bending resistance in the terminal filament. The best results were obtained by using values of $0.6,0.4,0.24,0.16$ and 0.08 times the value of $E_{B}$ used for the $9+2$ region of the flagellum.

A computer simulation was also run with values of $E_{B}$ equal to half of those used for Fig. 13. In this case, the increased curvature in the terminal filament was more extreme, and the results resembled those in Fig. I2 (data not shown). These values for the stiffness of the terminal filament appear to be too low. Another computer simulation was run with values of $E_{B}$ in the terminal filament equal to twice the values used for the computation in Fig. I3. In this case, there was no increase in curvature in the terminal filament, but there was a weak end effect of the type seen in Figs. 10 and I I (data not shown). These values for the stiffness of the terminal filament appear to be too high.

\section{DISCUSSION}

\section{Structure of the terminal filament}

The flagella of $C$. intestinalis and $L$. pictus have the usual $9+2$ axonemal structure, as shown in previous studies of tunicate (Schabtach \& Ursprung, 1965; Woollacott, 1977) and sea-urchin (Afzelius, 1955, 1959) spermatozoa. Our observations on the ultrastructure of the terminal filament confirm earlier observations indicating that the terminal filament contains a subset of the $9+2$ axonemal microtubules, covered by an extension of the flagellar membrane.

The earliest electron micrographs showing the terminal filament appear to be those of Lowman (1953), who examined shadow-cast preparations of salmon spermatozoa. The terminal filament appeared to contain two regions of decreased diameter: a basal region with a diameter of about $0.10 \mu \mathrm{m}$ and a length of about $\mathrm{I} \mu \mathrm{m}$, and a terminal region with a diameter of $0.03-0.06 \mu \mathrm{m}$ and a length of $\mathrm{I}^{1} 5^{-2} \mu \mathrm{m}$. Micrographs of disrupted flagella showed four microtubules in the basal region and two microtubules in the terminal region, but identification of central-pair microtubules was not possible.

A low-magnification scanning electron micrograph of an oyster spermatozoon, presented by Daniels, Longwell, McNiff \& Wolfgang (1971), appeared similar, with the basal region of the terminal filament having a length of about $\mathrm{I} \mu \mathrm{m}$ and the distal region a length of about $3 \mu \mathrm{m}$. A cross-section, identified as through the terminal filament, had a diameter of $0.14 \mu \mathrm{m}$ and showed two central microtubules within a ring of nine singlet microtubules, with no arms or spokes present. The size of this crcss-section suggests that it must be a section through the transition region of the terminal filament rather than through a more distal region.

Afzelius (1955) described cross-sections through the terminal filament of sea-urchin sperm flagella, identified by their reduced diameter (described as an ellipse with a 
major diameter of $0.09 \mu \mathrm{m}$ ). The sections showed numerous single microtubules, commonly with just two microtubules. In a later paper using improved fixation procedures, Afzelius (1959) described several types of cross-sections considered to be cross-sections of the terminal filament of sea-urchin sperm flagella. The typical appearance of sections considered to represent the major portion of the terminal filament was illustrated by a section showing five or six single microtubules, all of which had slightly smaller diameters than central-pair microtubules seen in other flagella in the same field. Two other types of sections, presumed to be closer to the $9+2$ region of the flagellum, are described. In one type, the central pair is surrounded by nine peripheral tubules, all (or most) of which are single. In the other type, the central pair is missing, and the peripheral doublets, although usually reduced in number, carry arms and spokes. The possibility that the central pair, known to be more labile from later work on other flagella, might be lost in the preparation of these sections was not examined.

The alga, Micromonas pusilla, was examined by Omoto \& Witman (198I), using negative staining and cross-sectioning techniques. This flagellum has a short $9+2$ region ( $<\mathrm{I} \mu \mathrm{m}$ in length), a very short transition region ( $0.2 \mu \mathrm{m}$ in length), and a terminal filament with a diameter of $0.08 \mu \mathrm{m}$ and a length of about $4 \mu \mathrm{m}$, which contains only the central pair of microtubules. A very similar terminal filament, containing a central pair of microtubules, was described for the flagellum of the fungus Rhizophidium (Koch, 1956). Koch (1956) also examined the fungus Chytridium, in which the terminal filament appears to be tapered more gradually, with three microtubules extending to the tip of the terminal filament. A description of the flagellum of the alga Monomastix by Manton ( 1967 ) indicates that only one microtubule extends through the length of the terminal filament.

In combination with our own observations on the terminal filaments of Ciona and Lytechinus sperm flagella, these reports suggest that the transition region of the terminal filament contains a variable number of microtubules. With the exception of the report by Afzelius (1959), these are always single microtubules devoid of dynein arms. Therefore, presumably, they lack any mechanism for active sliding or bending. The longer, major portion of the terminal filament usually contains the central pair of microtubles, and may also contain additional single microtubules, which are extensions of the A tubule of outer doublets. From this structural information, we might conclude that a precise structural pattern or a consistent microtubular complement is not important for the functioning of the terminal filament.

\section{The end effect and the effect of the terminal filament}

The end effect seen in the absence of the terminal filament, both in real flagella (e.g. Figs. 4B, 8B) and in computer simulations (Fig. 10), involves a rapid unbending of a bend that has reached the distal end of the flagellum. This end effect can be understood in terms of the balance of bending moments on the flagellum. It is easiest to explain with reference to a hypothetical bending wave composed of circular arcs (Brokaw, 1965). A shear curve for such a bending wave is illustrated in Fig. ${ }_{4} \mathrm{~A}$.

Consider first the static situation, in which there are no moments resulting from 
movement against viscous drag forces. In this situation, elastic bending moments must be balanced by active bending moments. If the elasticity of the flagellum is governed by an elastic bending resistance, $E_{B}$, and a region of the flagellum is bent into a circular arc of constant curvature, $\kappa$, there must be a constant bending moment equal to $E_{B} \kappa$ throughout the bent region. Active shear moments are required in the interbend region to obtain these bending moments and balance the elastic bending resistance of the flagellum (Fig. ${ }_{4} B$ ). The inclusion of elastic shear resistance, $E_{S}$,

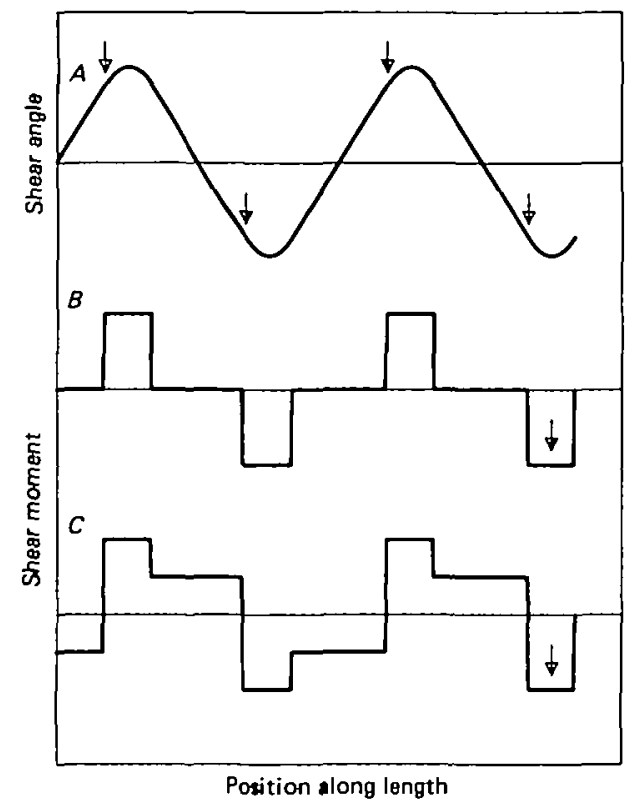

Fig. 14. Diagrams to illustrate the discussion of the end effect. $A$ is a shear curve for a hypothetical bending wave constructed of circular arcs (corresponding to the sloping lines) separated by transition regions in which there is a linear change in curvature with length. $B$ illustrates the distribution of active shear moment needed to maintain the static configuration of the bending wave represented in $A$, by balancing active and elastic bending moments. These distributions are approximate, and neglect the effect of elastic shear resistances. $C$ illustrates the approximate distribution of active shear moment needed to propagate the bending wave represented in $A$. This distribution is the result of adding active moment required to overcome viscous forces to $B$.

in the elastic resistance of the flagellum will modify this required distribution of active shear moment, but will not change the conclusion that the active shear moment is required primarily in the interbend regions.

In the dynamic situation, where bends are being propagated along the flagellum, additional active moment is required to overcome viscous resistances. These active shear moments are primarily required in the bent regions, which are also the regions of active sliding (Brokaw, 197I). The necessary distribution of active shear moments will in this case be more like that illustrated in Fig. ${ }_{4} C$. An approximation to this distribution of active shear moment is generated by the cross-bridge system incorporated into our computer simulations, as a result of two features of our flagellar 
model: (1) switching between two antagonistic cross-bridge systems on either side of the flagellum is accomplished when a critical curvature is reached, at points corresponding approximately to the arrows in Fig. ${ }_{4} A$; and (2) the active shear moment in a region of sliding is less than in an interbend region where the sliding velocity is low, because of the 'force-velocity' relationship that is characteristic of cross-bridge models for muscle and flagella (Huxley, 1957).

From these considerations, it should be clear that the active shear moment in the terminal interbend region, indicated by the arrows in Fig. ${ }_{4} B$ and $C$, is required to balance the elastic bending moment in the bend closest to the end of the flagellum. As this interbend region is propagated past the end of the flagellum, there will be insufficient active shear moment to balance the elastic bending moment, and the elastic moment will cause the distal bend to unbend. As shown by Fig. ${ }_{4} C$, the active shear moments generated within the distal bend will be unable to maintain this bend, because they are in the wrong direction. However, the viscous forces acting on the distal end of the flagellum will be in the direction that tends to maintain bending, and these will be opposed by the elastic bending moment in the distal bend as it unbends. Thus the balance between viscous forces and elastic bending moment will determine the rate of unbending.

When a terminal filament is present, the additional viscous drag that it introduces at the end of the flagellum will retard the unbending of a distal bent region when it reaches the point where no active shear moment is available to maintain its bent configuration. This provides a simple explanation of the effect of the terminal filament in eliminating the end effect. However, the detailed bending behaviour of the terminal filament cannot be explained simply in qualitative terms, and requires analysis by computer simulation.

This interpretation of the bending behaviour of the distal end of a flagellum is essentially the same as that suggested by earlier observations (Brokaw, 1965). However, those observations were based on the observation of end effects with much shorter flagellar fragments, where the unbending of bends at the end of the flagellum could not be separated from the unbending that occurs as bends enlarge during bend development at the basal end of the flagellum (Brokaw, 1970; Goldstein, 1976).

\section{Bending behaviour and stiffness of the terminal filament}

Among the computer simulations shown in this paper, the specification for the elastic bending resistance of the terminal filament used for the computation illustrated in Fig. 13 gives the best agreement with the observations on the bending behaviour of the sperm terminal filament. On the basis of the computations performed, we conclude that a precise match between any observed bending behaviour and the behaviour of a computer model could be obtained by careful refinement of the specifications for the elastic bending resistance of the terminal filament; specifically, the precise gradation of bending resistance along the length of the terminal filament. If the elastic bending resistance of microtubules is constant, we might expect the elastic bending resistance of the terminal filament to be proportional to the number of microtubules present at any position along its length. On the basis of our structural observations, we might 
then expect that the elastic bending resistance of the terminal filament would fall in between the specifications used for the computations in Fig. 12 and Fig. I3. Better results were obtained from computations in which the terminal filament was given a more tapered elastic bending resistance, corresponding more closely to the structural observations on Lytechinus spermatozoa.

The computations to model the Ciona sperm movement suggest that a reasonable value for the elastic bending resistance, $E_{B}$, in the major portion of the terminal filament is $0.03 \times 10^{8} \mathrm{pN} \mathrm{nm}^{2}$. This region of the terminal filament typically contains three microtubules, suggesting that an estimate for the elastic bending resistance of a single microtubule is $0.01 \times 10^{9} \mathrm{pN} \mathrm{nm}^{2}$. There is an uncertainty of at least a factor of 2 in this estimate. On the basis of this estimate for the stiffness of a single microtubule, a $9+2$ axoneme might be expected to have a stiffness of $0.20 \times 10^{9} \mathrm{pN} \mathrm{nm}^{2}$, as we used for the computer simulations. However, this extrapolation assumes that the stiffness of an axonemal doublet microtubule is equal to the stiffness of two single microtubules, and there is no independent evidence for this assumption.

This estimate for the value of $E_{B}$ for a $9+2$ flagellum has a simpler basis than previous estimates, since it depends only on the balance between elastic bending resistance and viscous drag forces in the terminal filament. $E_{B}$ can also be estimated by adjusting its value in the computer simulations until bending waves of realistic size are obtained, but this procedure depends strongly on the parameters chosen for the dynein cross-bridge system that generates active shear moment and the manner in which this system is controlled; it may also depend on the values used for the elastic shear resistance of the flagellar model. Information about $E_{B}$ has also been obtained from direct measurements of the bending resistance of sperm flagella (Okuno \& Hiramoto, 1979; Okuno, 1980). However, in these measurements, the measured bending resistance, equivalent to approximately $\mathrm{I} \cdot 0 \times 10^{9} \mathrm{pN} \mathrm{nm}^{2}$, also contains a significant contribution from the elastic shear resistance, since the method requires bending the flagellum under conditions where the curvature is low (comparable to a long 'wavelength') (cf. Blum \& Hines, 1979).

Using our calculated value for $E_{B}$ of $0.20 \times 10^{9} \mathrm{pN} \mathrm{nm}^{2}$ and Okuno's bending resistance measurement of $1 \cdot 0 \times 10^{9} \mathrm{pN} \mathrm{nm^{2 }}$, which includes components of shear and bend resistances, we estimate the elastic shear resistance of the axoneme to be $6.8 \mathrm{pN}$.

\section{Function of the terminal filament}

Since terminal filaments of approximately similar structure have been found at the distal ends of flagella from many different species, it is reasonable to assume that the terminal filament is functionally significant in improving the propulsive effectiveness of the flagellum. An increase in propulsive effectiveness could arise in two ways: by elimination of the end effect and by an increase in the effective length of the flagellum. We can predict that the effect of an increase in the effective length of the flagellum will be small. The velocity of an isolated flagellum (lacking a head) will be independent of the length, and the velocity of a spermatozoon with a small head is close to the limiting velocity for an isolated flagellum (Gray \& Hancock, 1955; Brokaw, 1965). A calculation using equation (I I) of Brokaw (1965), which gives the forward 
velocity for a flagellum propelling a spherical head, using resistive force theory (Gray $\&$ Hancock, 1955 ), indicates that for a spherical head of radius $2 \mu \mathrm{m}$, the increase in propulsive velocity associated with an increase in length of the flagellum from $50 \mu \mathrm{m}$ to $55 \mu \mathrm{m}$ would be less than I \%. This calculation assumes that addition of the terminal filament to the distal end of the flagellum causes no change in the parameters of movement of the flagellum. However, with our computer model a significant decrease in beat frequency, of more than $5 \%$, was observed when a terminal filament was added. Such a change would be sufficient to eliminate any advantage resulting from the increased length of the flagellum.

No analysis of the effect of an end effect, such as we have described here, on the propulsive effectiveness of a flagellum is available. We cannot examine this question simply by computing the swimming velocity of flagellar models with and without a terminal filament, because we do not know whether the response of the computer model to the increased drag resulting from addition of a terminal filament is the same as the response of a real flagellum. The question of relative propulsive effectiveness could, in principle, be answered by measuring the swimming velocities of spermatozoa with and without a terminal filament. However, because the effect appears to be small, and because there is considerable variation in swimming parameters within a population of spermatozoa, very large samples of spermatozoa would have to be compared under identical conditions. Even then, we could not be sure that the shearing used to remove the terminal filament did not have some additional effect that was expressed by a change in swimming velocity.

This question could be answered partially by hydrodynamic analysis using computational methods similar to those of Brokaw (1970) to calculate the swimming velocity for two flagellar waveforms that were identical except for the presence of an end effect. This analysis is beyond the scope of the present paper. Even this analysis will not answer the question of whether the combination of the hydrodynamic effects of eliminating the end effect and increasing the effective length of the flagellum, and the response of the flagellar mechanisms for generating bending waves to the altered viscous loading introduced by the presence of a terminal filament, will result in an increase in propulsive effectiveness. Therefore, the question of the function of the terminal filament remains unanswered.

We thank Sandee Nakada and Jean Edens for assistance in the laboratory work, Dr J.-P. Revel for advice on electron microscopy and Dr H. Berg and Markus Meister for comments on an earlier version of this manuscript. This work has been supported by grants GM 18711 and GM 21931 to C.J.B. and F32-GMo7445 to C.K.O.

\section{REFERENCES}

AfZelius, B. (1955). The fine structure of the sea urchin spermatozoa as revealed by the electron microscope. Z. Zellforsch. mikrosk. Anat. 42, 1 34-148.

Afzelius, B. (1959). Electron microscopy of the sperm tail. 7 . biophys. biochem. Cytol. 5, 269-278. Blum, J. J. \& Hines, M. (1979). Biophysics of flagellar motility. Q. Rev. Biophys. 12, 103-180. Brokaw, C. J. (1965). Non-sinusoidal bending waves of sperm flagella. f. exp. B.ol. 43, I 55I69.

Brokaw, C. J. (1970). Bending moments in free-swimming flagella. J. exp. Biol. 53, 445-464. 
Brokaw, C. J. (1971). Bend propagation by a sliding filament model for flagella. F. exp. Biol. $55,289-304$.

Brokaw, C. J. (1972). Flagellar movement: a sliding filament model. Science, N.Y. 178, 455462.

Brokaw, C. J. (1979). Calcium-induced asymmetrical beating of Triton-demembranated sea urchin sperm flagella. 7 . Cell Biol. 82, 401-411.

BrokAw, C. J. (1980). Theoretical models for oscillation and bend propagation by sperm flagella. In Testacular Developement, Structure and Function (ed. A. Steinberger \& E. Steinberger), pp. 447-453. New York: Raven Press.

BroKaw, C. J. (1982a). Generation of the bending cycle in cilia and flagella. Cell Motil. (8uppl.) I, I37-141.

Brokaw, C. J. ( $982 b$ ). Models for oscillation and bend propagation by flagella. Symp. Soc. exp. Biol. 35, 31 3-338.

Brokaw, C. J. (1982c). Activation and reactivation of Ciona spermatozoa. Cell Motil. (suppl.) I, I 85-189.

Brokaw, C. J., Luck, D. J. L. \& Huang, B. (1982). Analysis of the movement of Chlamydomonas flagella. The function of the radial spoke system is revealed by comparison of wildtype and mutant flagella. F. Cell Biol. 92, 722-732.

Brokaw, C. J. \& Rintala, D. (1975). Computer simulation of flagellar movement. III. Models incorporating cross-bridge kinetics. J. Mechanochem. Cell Motil. 3, 77-86.

Daniels, E. W., Longwell, A. C., MCNiff, J. M. \& WolfGaNG, R. W. (I971). Ultrastructure of spermatozoa from the American oyster Crassostrea virginica. Trans. Am. microsc. Soc. 90, 275-282.

Gibbons, I. R. (198I). Transient flagellar waveforms during intermittent swimming in sea urchin sperm. II. Analysis of tubule sliding. F. Muscle Res. Cell Motil. 2, 83-1 30.

GolDSTEIN, S. F. (1976). Form of developing bends in reactivated sperm flagella. f. exp. Biol. 64, $173-184$

Goldstein, S. F. (1977). Asymmetric waveforms in echinoderm sperm flagella. $\mathcal{Y}$. exp. Biol. 71, $157-170$.

GRAY, J. (1955). The movement of sea urchin spermatozoa. F. exp. Biol. 32, 775-801.

GrAY, J. \& HANCOCK, G. J. (1955). The propulsion of sea urchin spermatozoa. f. exp. Biol. 32, $802-8$ I 4 .

Hines, M. \& Blum, J. J. (1978). Bend propagation in flagella. I. Derivation of equations of motion and their simulation. Biophys. f. 23, $4 \mathrm{I}-58$.

HiNes, M. \& BLUM, J. J. (1979). Bend propagation in flagella. II. Incorporation of dynein cross-bridge kinetics into the equations of motion. Biophys. F. 25, 421-44I.

Hiramoto, Y. \& BABA, S. A. (г978). A quantitative analyBis of flagellar movement in echinoderm spermatozoa. F. exp. Biol. 76, 85-104.

HuXLEY, A. F. (1957). Muscle structure and theories of contraction. Prog. Biophys. 7, 255-318.

Jarosch, R. \& FuCHS, B. (1975). Zur fibrillenrotation in der Symura-Geissel. Protoplasma 85, 285-290.

Johnson, R. E. \& Brokaw, C. J. (1979). Flagellar hydrodynamics. A comparison between resistive-force theory and slender-body theory. Biophys. J. 25, i i 3-I 27.

Johnston, D. (1978). Theoretical and experimental aspects of flagellar wave shape analysis and their application to the protozoan, Crithidia oncopelti. Ph.D. thesis, University of London.

Johnston, D., Silvester, N. R. \& Holwill, M. E. J. (1979). An analysis of the shape and propagation of waves on the flagellum of Crithidia oncopelti. J. exp. Biol. 80, 299-3 5 .

KamiYa, R., Nagai, R. \& Nakamura, S. (1982). Rotation of the central-pair of microtubules in Chlamydomonas flagella. In Biological Functions of Microtubules and Related Structures (ed. H. Sakai, H. Mohri \& G. G. Borisy) London: Academic Press (In Press).

KELleR, J. B. \& Rubinow, S. I. (1976). Slender-body theory for slow viscous flow. F. Flitid Mech. 75, 705-714.

Koch, W. J. (1956). Studies on the motile cells of Chytrids. I. Electron microscope observations of the flagellum, blepharoplast and rhizoplast. Am. F. Bot. 43, $8 \mathrm{I}$ I-8Ig.

Lighthill, J. (1976). Flagellar hydrodynamics. Soc. Ind. appl. Math. Rev. 18, 16 I-230.

Lowman, F. G. (1953). Electron microscope studies of silver salmon spermatozoa (Oncorhynchus kisutch). Expl Cell Res. 5, 335-349. 
Manton, I. (I967). Electron microscopical observations on a clone of Monomastix Scherffel in culture. Nova Hedroigia 14, $1-11$.

OKuno, M. (1980). Inhibition and relaxation of sea urchin sperm flagella by vanadate. $\%$. Cell Biol. 85, 712-725.

Okuno, M. \& Hiramoto, Y. ( 1979 ). Direct measurements of the stiffness of echinoderm sperm flagella. F. exp. Biol. 79, 235-243.

Oмото, C. K. \& Witman, G. B. (1981). Functionally significant central-pair rotation in a primitive eukaryotic fagellum. Nature, Lond. 290, 708-710.

RikMeNSPOEL, R. (1978). Movement of sea urchin sperm flagella. f. Cell Biol. 76, 310-322.

Schabtach, E. \& Ursprung, H. (1965). The fine structure of the sperm of a tunicate Ascidia nigra. F. exp. Zool. 159, 357-366.

SOMMER, J. R. (1977). To cationize glass. F. Cell Biol. 75, 245 a.

Woollacott, R. M. (1977). Spermatozoa of Ciona intestinulis and analysis of Ascidian fertilization. Y. Morph. r52, 77-88.

(Received 6 May 1982) 
